\title{
Photo-Triggering of the Membrane Gates in Photo-Responsive Polymer for Drug Release
}

\author{
Jae Kyoo Lee ${ }^{1,4}$, Hoeun Lee ${ }^{2}$, Eunje Jang ${ }^{3}$, Sin-Doo Lee ${ }^{3}$, and Sung June Kim ${ }^{1,3,4}$ \\ ${ }^{1}$ Interdisciplinary Program of Biomedical Engineering, Seoul National University, Seoul, Korea \\ ${ }^{2}$ School of Chemical and Biomolecular Engineering, Cornell University, Ithaca, New York \\ ${ }^{3}$ School of Electrical Engineering, Seoul National University, Seoul, Korea \\ ${ }^{4}$ Nano-Bioelectronics and Systems Research Center, Seoul National University, Seoul, Korea
}

\begin{abstract}
The use of light stimulus for triggering drug is a promising method for accurate drug delivery. A new approach using azopolymer membrane and laser holography was investigated for developing light-triggering drug delivery system. Polymeric drug delivery system was prepared by covering azopolymer membrane on a drug agent. Holographic laser interference generated surface relief grating pattern on the azopolymer surface. The widths and depths of gates on the polymer membrane were easily modified by adjusting incident angle and irradiation time. Ar laser made the polymeric membrane permeable to the drug agent and release it in a solution. This result indicated that the azopolymer and laser holography would provide a strong foundation for the light-triggering drug delivery system.
\end{abstract}

\section{INTRODUCTION}

Site-specific and time-dependant triggering of drugs is a focused area in developing drug delivery systems. Various methods of stimuli for the environment-sensitive drug delivery have been proposed, including $\mathrm{pH}$, temperature, pressure, biochemical, light, electronic, and magnetic stimuli [1]. Since the light stimulus can be imposed upon a target with high accuracy and specific amount of drugs, light-controlled drug delivery system possesses high advantages over others [2]. Most of the light-triggering methods utilize photostimulated cis-trans isomerism, photoinduced lipid fragmentation, lipid photopolymerization, and photothermal phenomena [3].

Azobenzene copolymer is a new class of optically active material responding to various wavelengths depending on the substituents on the benzene ring. Azobenzene molecules tend to orient parallel to the direction of polarization when exposed to polarized light. Interfering two orthogonal lights on the surface of the polymer induces a massive migration of the molecules and consequent micro and submicro structures of repeated grooves [4], [5].

This surface relief grating (SRG) is an easy and simple method of modulating permeability of polymeric film. Polarization-dependant molecular migration produces the changes in membrane thickness and opening of polymeric gates through azopolymer film. Modifying the interference angle and polarization allows controlling the gating through azopolymer membrane. By modulating the gate size and permeability, the release rate and amount of drug imposed to tissue and cells can be controlled. Since the light-induced deformation of azopolymer is reversible and repeatable [6], the opened gate can be closed to control the amount of drug release. The aim of this study is to develop a new method for controlling the permeabilization of polymeric membrane by holographic SRG using visible laser light.

\section{MATERIALS AND METHODS}

\section{A. Photoresponsive Polymer and Preparation of Drug Delivery System}

Fluorescence dye (excitation 350nm) was used as a dug agent for the evaluation of triggering dugs. Fluorescence dye was diluted in phosphate-buffered saline (PBS) and coated on glass substrate. The agents were completely dried before polymer film was coated to prevent mixing of the drug liquid and the solvent of polymer. Azobenzene copolymer, poly [(methylmethacrylate)-co-(disperse red 1 acrylate)] (57042-7, Sigma Aldrich) was used as a photoresponsive polymer. It was dissolved in tetrahydrofuran at a concentration of $4 \%$ (w/w). The polymer film was formed by spin coating on a cover glass, which produced a coated film of a thickness of about $500 \mathrm{~nm}$ (Fig. 1). The coated polymer was dried for 6 hours at $70^{\circ} \mathrm{C}$ to remove the solvent. 
Polymer Coating
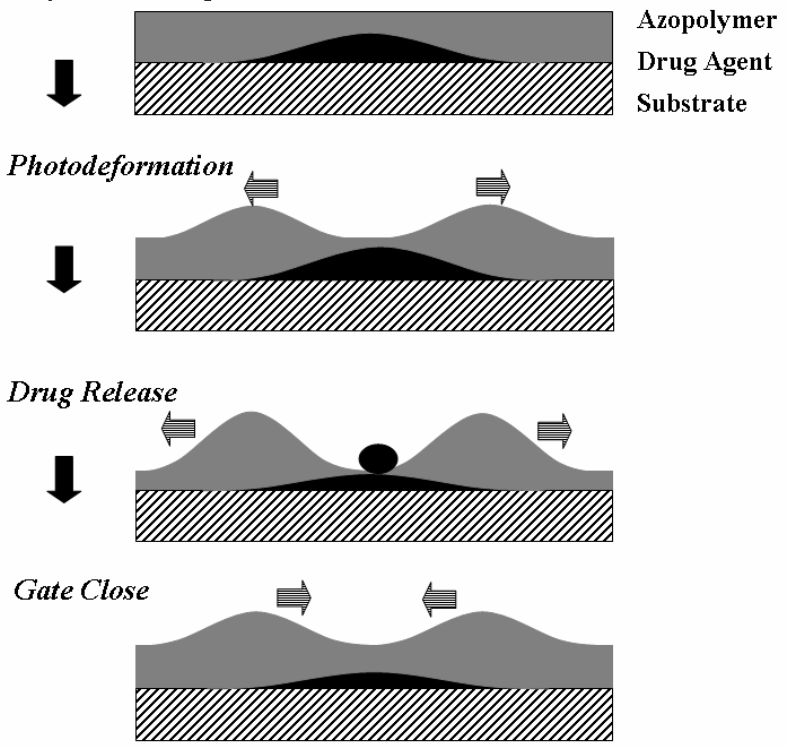

Fig. 1. Schematic view of triggering and closing of drug release. Azopolymer film was coated on drug agents and deformed by polarized laser irradiation. Opened polymeric gate on azopolymer film was closed by modulating the direction of polarization.

\section{B. Surface Relief Grating}

In order to form a holographic SRG, we used a 488-nm $\mathrm{Ar}^{+}$ ion laser and the classic Lloyd's mirror setup (Fig. 2). Light from the laser was expanded by a beam expander at an appropriate range and polarized. We formed an interference pattern by the superposition of two beams. One came directly from the laser and the other was reflected from a mirror. The incident beam from the laser was linearly polarized at an angle of $+45^{\circ}$ while the reflected beam from the mirror had an angle of $-45^{\circ}$ with respect to the substrate normal. These two orthogonal beams exhibited polarization modulation on the polymer surface resulting in molecular migration to form regular sinusoidal SRG. The intensity of $\mathrm{Ar}^{+}$ion laser was about $300 \mathrm{~mW} / \mathrm{cm}^{2}$. The width of grooves was determined by the combination angle between the two beams, and the depth by the duration of laser irradiation. The formation of the grating was monitored in real time by probing with a $\mathrm{He}-\mathrm{Ne}$ laser (wavelength of $633 \mathrm{~nm}$ ) onto the inscribed region. The probe beam was linearly polarized at $45^{\circ}$ to the axis of grating and did not influence the deformation process.

\section{Measurements of Fluorescence and Surface Characteristics}

Spectrofluormeter (JASCO, FP-6500) and fluorescence microscope (Carl Zeiss, LSM 120) were used to observe the released fluorescence dye in PBS solution. The observations

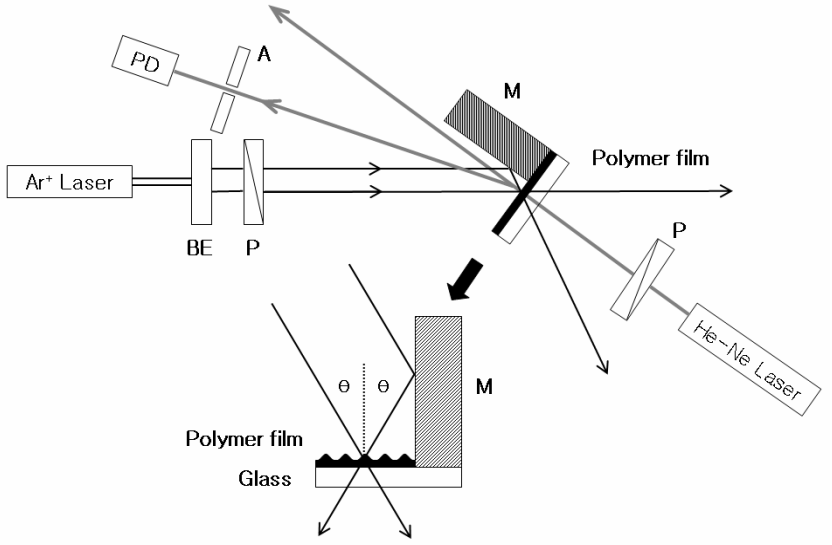

Fig. 2. Schematic view of the optic setup for SRG formation. The laser beam was linearly polarized at an angle of $+45^{\circ}$ and the reflected beam on the mirror had an angle of $-45^{\circ}$ with respect to the substrate normal. Two orthogonal beams interfered on the surface of the polymer to form the groove structure. The period of the grating pattern depended on the combination angle $\theta$. (PD: photo detector, BE: beam expander, P: polarizer, M: mirror, A: aperture)

were performed both before and after treating laser irradiation on azopolymer film with fixed excitation wavelength of $350 \mathrm{~nm}$. After inscribing SRG on the polymers, the exposed surfaces were investigated with an atomic force microscope (AFM, XE-150, PSI) in contact mode.

\section{RESULTS AND DISCUSSION}

\section{A. Light-induced Photodeformation}

A deformation of azopolymer surface was observed using AFM scanning in Fig. 3. The interference between two orthogonally polarized beams made polarization modulation on the surface of the polymer. As a result, deep grooved patterns were inscribed on the azopolymer film. The depths of the grooves were determined by the duration of laser irradiation, and the widths by the angle of the incident beam. The He-Ne laser revealed that the intensity of the diffracted probe beam increased with the groove depth, and the end point of deformation was estimated by measuring the relative intensity of the diffracted beam. The ATM revealed an absence of debris on the surface of the polymer after fabricating the SRG. The surface topography was quite homogeneous in the laser-irradiated area, confirming the success of the membrane deformation technique. The maximum depth of SRG from the experiments reached up to $800 \mathrm{~nm}$ and the width ranged between hundreds of $\mathrm{nm}$ and 6 $\mu \mathrm{m}$, which were sufficient to make the film permeable to drugs. 


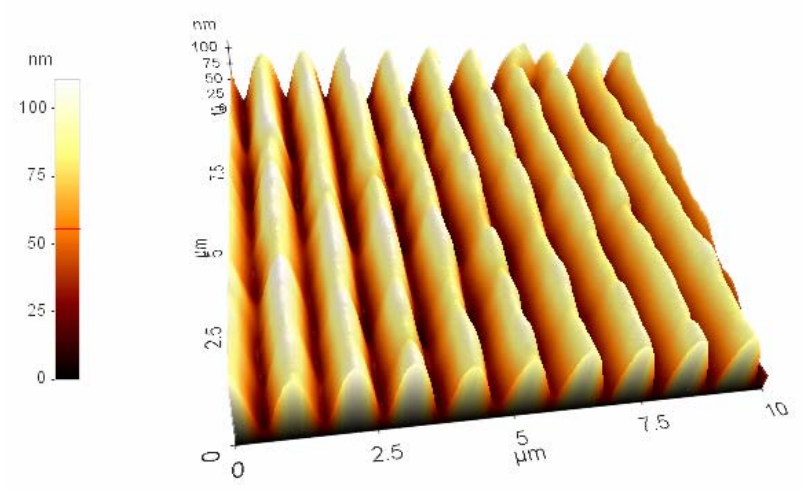

Fig. 3. AFM images of SRG produced by laser holography of the surface of the azobenzene copolymer. The widths and depths of grating structure were $1.2 \mu \mathrm{m}$ and $110 \mathrm{~nm}$.

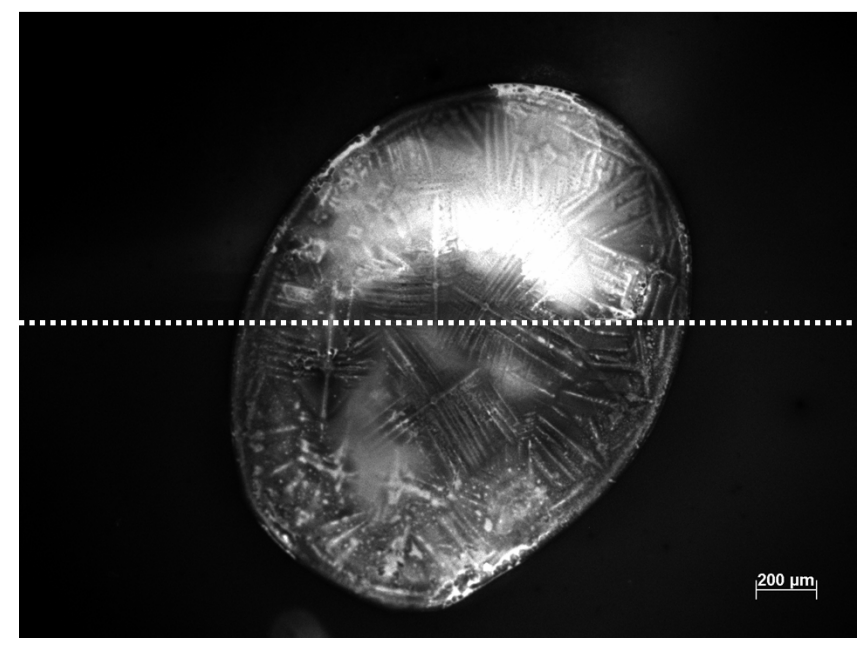

Fig. 4. Fluorescence image of a sample. SRG (1 $\mu \mathrm{m}$ wide, $600 \mathrm{~nm}$ deep) are present only above the white dotted lines. Fluorescence dye was released in the upper region and kept in the polymeric membrane in the lower region.

\section{B. Drug Release Test}

The samples were prepared by the procedure presented in Fig. 1. A fluorescence dye was coated on glass substrate and covered by spin-coated azopolymer film. The thicknesses of the polymer film were $500 \mathrm{~nm}$ in the region without fluorescence dye and $200 \mathrm{~nm}$ in the region with fluorescence dye. The samples were irradiated by holographic interference pattern of Ar laser. After being treated with Ar laser, a sample was dipped into a Petri dish containing PBS and analyzed by fluorescence microscope. Figure 4 shows the fluorescence image of the sample. Dye existed in the middle white area in circle and covered by azopolymer film. Polymeric gates were opened by forming SRG only above the white dotted lines. It was observed that fluorescence dye was released in region where the SRGs were formed. In other regions, the dye was confined in the

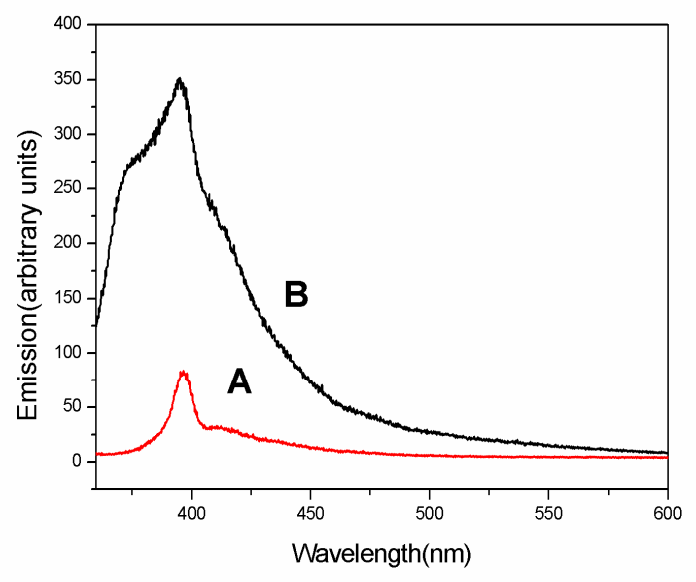

Fig. 5. Plot of emission from sample before treating laser (A) and after (B) measured by spectrofluormeter.

polymeric membrane and not highlighted. The crystalline structure in the region where the dye existed was due to the crystallization of ingredients from PBS.

Samples containing fluorescence dyes were irradiated by Ar laser for opening gates through polymeric membrane. For the release tests conducted, spectrofluormeter plot of emission intensity against wavelength was presented in Fig. 5. After releasing drugs, it was observed that the intensity of fluorescence in PBS was increased by approximately five times. The released polymeric gates can be closed to prevent an additional drug infusion into target cells of tissue. By modulating the polarization state of the erasing beam, the inscribed structure on the azopolymer is erased and polymer membrane is recovered

\section{CONCLUSION}

Light-triggered drug delivery has been explored for the potential application in ophthalmic drug delivery. Azopolymer membrane containing a drug agent was prepared for the controlled drug releasing. Laser holography of $\mathrm{Ar}$ laser deformed the surface of azopolymer and produced regular grooves pattern. The test of drug triggering showed that fluorescence dye was released in the laser treated region and kept in polymeric membrane. This in vitro study proved that the azopolymer membrane and laser holographic drug-triggering are capable of being used to controlled drug release system.

\section{ACKNOWLEDGMENT}

This work was supported by Korea Science and Engineering Foundation (KOSEF) through Nano Bioelectronics and Systems Research Center (NBS-ERC) in Seoul National University. 


\section{REFERENCES}

[1] Y. Qiu, K. Park, "Environment-sensitive hydrogels for drug delivery," Adv. Drug Deliv. Rev., vol. 53, no. 3, pp. 321 339, Dec. 2001.

[2] 2. T. Spratt, B. Bondurant, and D. F. O'Brien, "Rapid release of liposomal contents upon photoinitiated destabilization with UV exposure," Biochim. Biophys. Acta., vol. 1611, pp 35 43, Apr 2003.

[3] 3. P. Shum, J. M. Kim, and D. H. Thompson, "Phototriggering of liposomal drug delivery systems," Adv. Drug Deliv. Rev., vol. 53, no. 3, pp. 273 284, Dec. 2001.

[4] 4. A. Natansohn and P. Rochon, "Photoinduced motions in azo-containing polymers," Chem Rev., vol. 102, no. 11, pp. 41394175 , Nov 2002.

[5] 5. O. Kulikovska, K. Gharagozloo-Hubman, and J. Stumpe, "Polymer surface relief structures caused by light-driven diffusion," Proc. SPIE, vol. 4802, no. 85, pp. 85 94, Nov. 2002.

[6] 6. X. L. Jiang, L. Li, J. Kumar, D. Y. Kim, and S. K. Tripathy, "Unusual polarization dependent optical erasure of surface relief gratings on azobenzene polymer films," Appl. Phys. Lett., vol. 72, no. 20, pp. 2502 2504, May 1998 